\title{
Low Sample Rate Transmitter for Direct-Detection Optical OFDM
}

\author{
Brendon J. C. Schmidt, Arthur J. Lowery and Liang B. Du \\ Department of Electrical \& Computer Systems Engineering, Monash University, Clayton, 3800, Australia \\ Tel: +61 39905 3486, Fax: +61 39905 3454, E-mail: arthur.lowery@eng.monash.edu.au
}

\begin{abstract}
We experimentally demonstrate a novel colorless transmitter for direct-detection optical OFDM that requires lower sample-rate DACs than previous designs, without RF mixers. We transmit 24-Gbit/s over 800-km SSMF using two $10 \mathrm{GS} / \mathrm{s}$ DACs.

(C)2009 Optical Society of America

OCIS codes: (060.2330) Fiber optics communications; (060.4080) Modulation.
\end{abstract}

\section{Introduction}

Over the last two years, optical Orthogonal-Frequency Division Multiplexing (OFDM) for long-haul dispersion compensation, has developed from early proposals backed by simulation [1], [2], to experimental systems [3]. Optical-OFDM can offer data rates in excess of $100 \mathrm{Gbit} / \mathrm{s}$ per WDM channel [4], [5].

Direct-Detection Optical OFDM (DDO-OFDM) [6] offers the possibility of far simpler optical receiver architectures than coherent optical OFDM (CO-OFDM) [2], and also has far-less stringent requirements on the laser linewidth [7]. We have previously demonstrated DDO-OFDM experimentally using three different transmitter configurations [3]. Of most interest for wavelength-agile networks is a 'colorless' transmitter design, where the transmitted wavelength is governed solely by the wavelength of the laser; rather than also being set by an optical filter within the transmitter [1]. Our colorless transmitter used a single-sideband optical modulator (a complex or IQ, optical modulator), which allows the inphase (I) and quadrature (Q) components of an electrical OFDM signal to be mapped to the I and Q components of a complex optical field. Unfortunately, this requires a high sample rate of the DACs: approximately twice the data rate for 4-QAM modulation because of the frequency gap used between the optical carrier and information-bearing band of optical subcarriers, used to mitigate subcarrier $\times$ subcarrier noise. One method to reduce the required sample rate is to shift the laser frequency in the opposite direction to the subcarriers, by using the most negative frequency as a 'virtual optical carrier' [8]; this wastes half the DACs' bandwidth if a gap is used. Another is to use high-bandwidth RF mixers to upconvert the baseband I and Q signals, but this places the signal band at the edge of the optical modulator's response.

In this paper we propose a method of reducing the DACs' sample rates, without using RF mixers, while retaining a colorless transmitter. The signal band is centered on DC, so a modulator with bandwidth $B$ will provide a modulation bandwidth of $2 B$, supporting a data rate of $4 B$ (4-QAM). We show experimental results of $16 \mathrm{Gbit} / \mathrm{s}$ using 4-QAM and $24 \mathrm{Gbit} / \mathrm{s}$ using 8-QAM, with a simple direct-detection receiver and 5-GHz bandwidth DACs.

\section{System Design}

Fig. 1 shows the system. The details are explained in previous papers, e.g. [1]. In previous designs, 75\% of the IFFT inputs were set to zero, to null negative frequencies and provide a gap between the optical carrier and the information-bearing sideband. The I and Q components of the output of the IFFT were directly fed the optical modulator. In this design, all of the IFFT inputs can carry data. The optical carrier is derived by shifting the laser frequency using a separately-generated RF carrier, added between the DACs and the modulator. A single photodiode can be used at the receiver, and the I and Q components can be recovered using an RF mixer and two analog to digital converters (ADCs). A cyclic prefix of $6.25 \%$ was used.

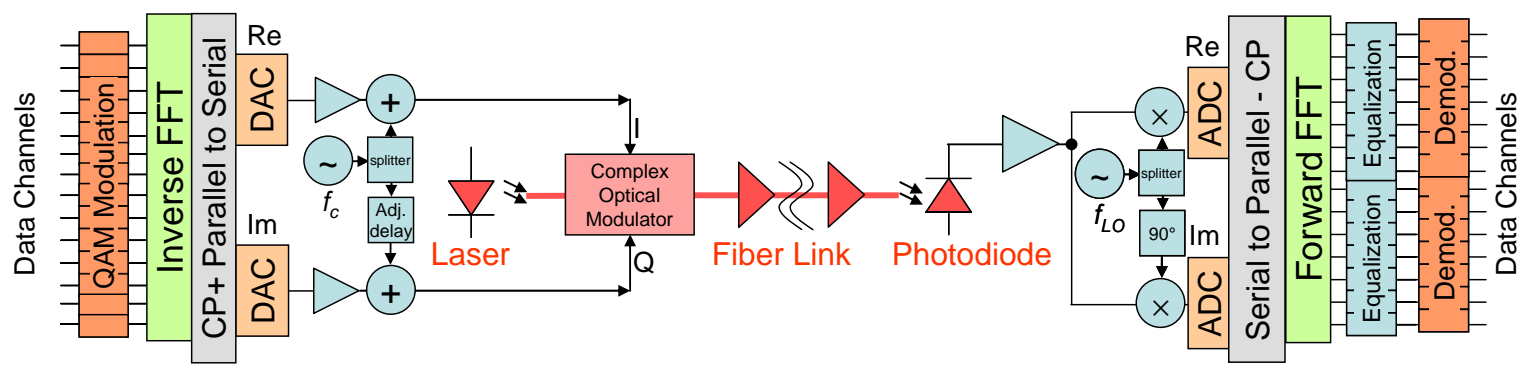

Fig. 1. Direct-detection OFDM system incorporating an RF generator at the transmitter. 


\section{OWM4.pdf}

Figure 2 compares the electrical and optical spectra for three colorless DDO-OFDM transmitters. Spectra A (left) are for the colorless transmitter proposed in [3]. Here, the electrical spectrum comprises a DC component and a subcarrier band. The DC component is used to bias the optical modulator, so provides an optical carrier, $f_{c a r}$, falling at the laser frequency, $f_{\text {laser }}$. Only half of the positive-frequency part of the electrical spectrum is used to convey data. Thus, the DACs sample at a rate $f_{\text {samp }}$, which must be at least 4-times the bandwidth of the subcarrier band, $B_{S C}$. Spectra B (center) are for the virtual optical carrier method [8]. Here, an RF tone is generated in the negative part of the electrical spectrum by applying appropriate inputs to the negative-frequency coefficients of the IFFT. The full positive-frequency part of the electrical spectrum carries data, so the required DAC sampling rate is halved. Spectra C (right) are for our proposed transmitter. Both the positive and the negative sides of the electrical spectrum are used to carry information, by applying data to the positive and negative coefficients of the IFFT. The positive and negative frequencies of the IFFT map directly onto the optical spectrum, centered around the laser line. The laser line itself is shifted to below the signal spectrum, to give an optical carrier. This shifting is achieved by applying sine and cosine waveforms to the complex optical modulator, generated by an RF oscillator, splitter and a phase shifter. This technique allows the sample rate to be reduced to the subcarrier bandwidth, $f_{\text {samp }}=B_{S C}$. No electrical mixers are required, because we are adding a high-frequency RF waveform, rather than frequency-shifting the subcarrier band. The strength and frequency of the optical carrier are simply set by the power and frequency of the RF waveform.

\section{DACs Output (Double-sided electrical spectrum)}
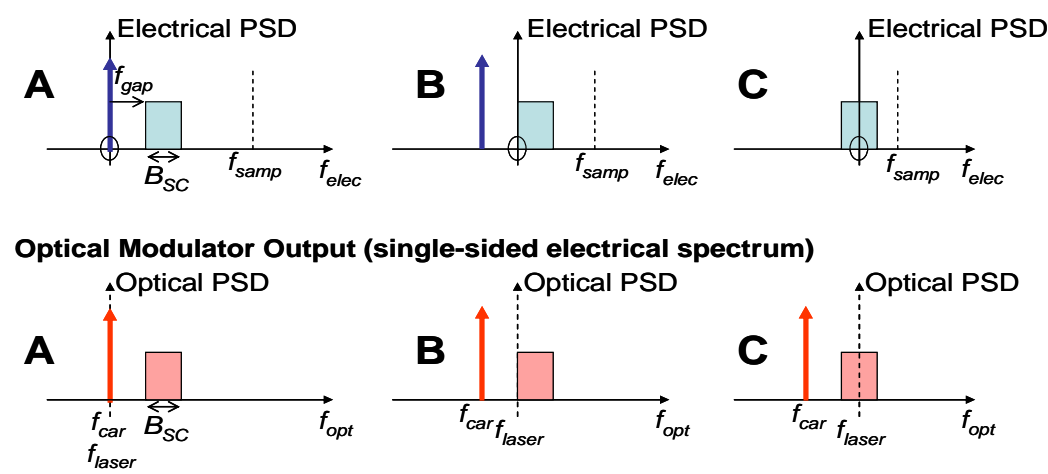

Fig. 2. Representative optical and electrical spectra from three types of direct-detection OFDM transmitter.

\section{Experimental Results}

The experimental system used a two-channel arbitrary waveform generator (AWG) with a DAC sampling rate of 10 GS/s to generate the OFDM's I and Q signals. These were amplified with Marki A-0200 microwave amplifiers and then combined with the I and Q components of an RF carrier. Microwave phase shifters were used to set the components of the RF signal in quadrature. A Sumitomo single-sideband optical modulator modulated a tunable laser. Ten 80-km spans of Corning SMF-28e fiber were preceded by Lightwave2020 optical amplifiers and followed by a preamplifier, an optical filter, a Discovery Semiconductors DSC-40R, and digitized with a Tektronix DSO72004. MATLAB was used for downconversion and processing. Two OFDM symbol formats were used: (a) 512 4QAM subcarriers with 2-times oversampling giving 5-GHz subcarrier bandwidth and $10 \mathrm{Gbit} / \mathrm{s}$; (b) 400 datacarrying subcarriers with 1.25-times oversampling giving an 8-GHz subcarrier bandwidth, supporting $16 \mathrm{Gbit} / \mathrm{s}$ with 4-QAM or $24 \mathrm{Gbit} / \mathrm{s}$ with 8-QAM.
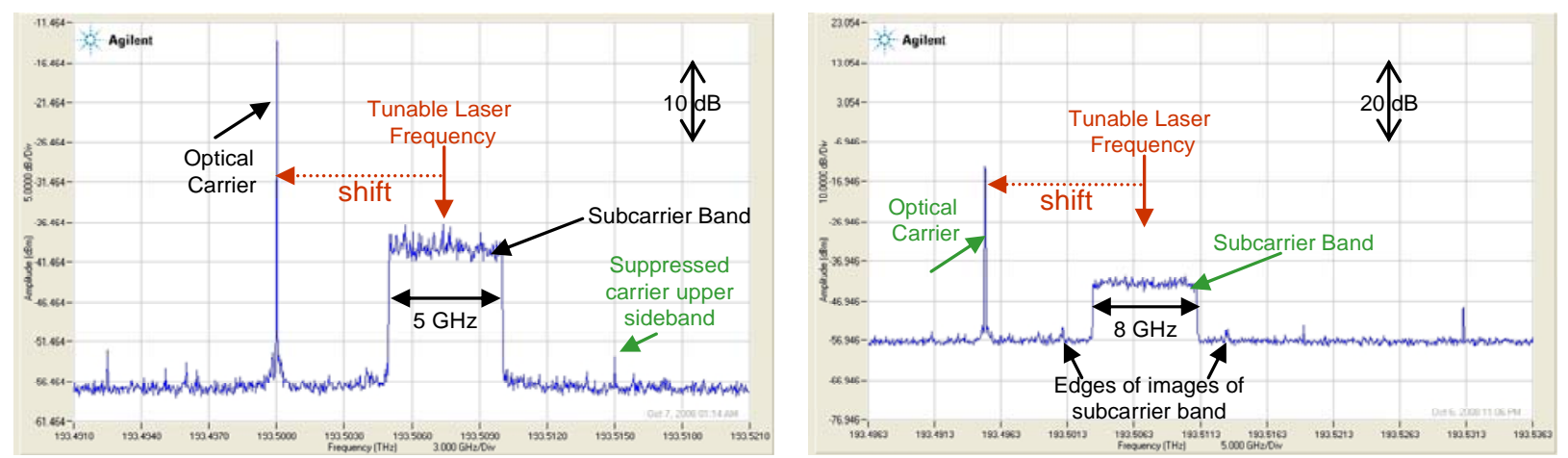

Fig. 3. Optical Spectra for the 5-GHz signal (left) and 8-GHz signal (right). 


\section{OWM4.pdf}

Fig. 3 shows the optical spectra for the two signal formats obtained using an Agilent High-Resolution Spectrophotometer. The carrier has been shifted downwards in frequency, as desired, to give a frequency gap between the carrier and the subcarrier band. The oversampling, in combination with analog filtering at the DAC outputs, highly suppresses the signal image, which can be barely seen in the right-hand figure. Note that the upper $\mathrm{RF}$ sideband and the laser carrier are also well suppressed in both figures.

Fig. 4 shows example constellations and a screenshot of the received time domain signal and electrical spectrum. Using ASE noise loading, for 4-QAM 16-Gbits/s we obtained a BER of $10^{-3}$ at OSNR $=12.5 \mathrm{~dB}$ (back-back, 20$\mathrm{GHz}$ filter); for 8-QAM 24-Gbps, 18.5-dB OSNR gave a BER of $2 \times 10^{-4}$ (800 km, 40-GHz filter). Narrower optical filters would improve the OSNR performance [9]. The RF spectrum shows the unwanted subcarrier $\times$ subcarrier mixing $(\mathrm{s} \times \mathrm{s})$ falling at frequencies below the wanted subcarrier $\times$ carrier $(\mathrm{s} \times \mathrm{c})$ products, because of the gap in the optical spectrum. Optimum performance is obtained when the powers in these two bands are approximately equal; adjusting the carrier level using the RF power level enables this condition to be easily met. The gaps in the waveform show the carrier level, noting the waveform has been inverted by the photoreceiver.
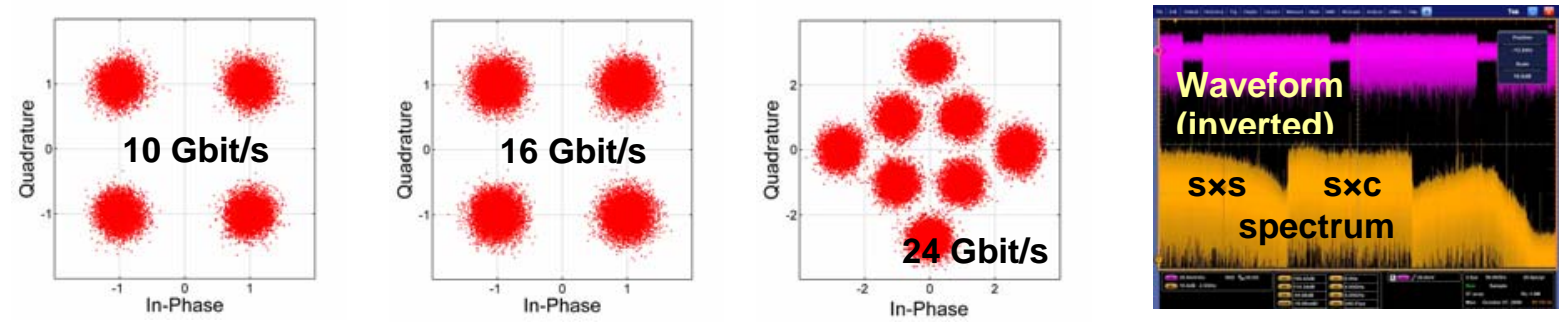

Fig. 4. Constellations for the three experiments (left): screenshot of the $24 \mathrm{Gbit} / \mathrm{s}$ waveform and RF spectrum (right). OSNR=18.5 dB.

\section{Conclusions}

We have presented a new design for a transmitter for direct-detection optical OFDM. This has the advantages of requiring reduced sampling-rate DACs for a given subcarrier bandwidth. Also, it does not require broadband RF mixers, optical modulators, or broadband RF power amplifiers. The optical carrier level and frequency are easily adjusted, to allow, for example, the use of carrier suppression and boosting or a reduced carrier gap. We demonstrate the system using commercial 10 GSamp/s DACs to give a 24-Gbit/s direct-detection OFDM system.

\section{Acknowledgements}

This research is supported under the Australian Research Council's Discovery funding scheme (DP0772937). We should like to thank VPIsystems.com for the use of VPItransmissionMaker for simulations leading to this design.

\section{References}

[1] A. J. Lowery and J. Armstrong, "Orthogonal frequency division multiplexing for dispersion compensation of long-haul optical systems," Optics Express, vol. 14, pp. 2079-2084, 2006.

[2] W. Shieh and C. Athaudage, "Coherent optical orthogonal frequency division multiplexing," Electronics Letters, vol. 42, pp. 587-588, 2006.

[3] B. J. C. Schmidt, A. J. Lowery, and J. Armstrong, "Experimental demonstrations of 20 Gbit/s direct-detection optical OFDM and 12 Gbit/s with a colorless transmitter," presented at Optical Fiber Communications, Anaheim, CA., paper PDP18, 2007.

[4] S. L. Jansen, I. Morita, and H. Tanaka, "10x121.9-Gb/s PDM-OFDM Transmission with 2-b/s/Hz Spectral Efficiency over 1,000 km of SSMF," presented at Optical Fiber communication/National Fiber Optic Engineers Conference, 2008. OFC/NFOEC 2008., San Diego, paper PDP2, 2008.

[5] W. Shieh, Q. Yang, and Y. Ma, "107 Gb/s coherent optical OFDM transmission over 1000-km SSMF fiber using orthogonal band multiplexing," Optics Express, vol. 16, pp. 6378-6386, 2008.

[6] A. J. Lowery, "Improving sensitivity and spectral efficiency in direct-detection optical OFDM systems," presented at Optical Fiber communication/National Fiber Optic Engineers Conference, 2008. OFC/NFOEC 2008. paper OMM4, 2008.

[7] A. J. Lowery, "Amplified-spontaneous noise limit of optical OFDM lightwave systems," Optics Express, vol. 15, pp. 860-865, 2008.

[8] P. Wei-Ren, W. Xiaoxia, V. R. Arbab, B. Shamee, L. C. Christen, Y. Jeng-Yuan, F. Kai-Ming, A. E. Willner, and C. Sien, "Experimental demonstration of a coherently modulated and directly detected optical OFDM system using an RF-Tone insertion," presented at Optical Fiber communication/National Fiber Optic Engineers Conference, 2008. OFC/NFOEC 2008, San Diego, CA, paper OMU2, 2008.

[9] A. J. Lowery, "Amplified-spontaneous noise limit of optical OFDM lightwave systems," Optics Express, vol. 16, pp. 860-865, 2008. 